\title{
Aspetti neurologici nella malattia di Anderson-Fabry
}

\author{
Antonello Giordano \\ UOC Neurologia, Presidio Ospedaliero "Riccardo Guzzardi", Vittoria (RG)
}

\begin{abstract}
Neurological aspects of Anderson-Fabry disease
Anderson-Fabry's disease involves both the central nervous system and peripheral nervous system, resulting in cerebrovascular disease, painful neuropathy, and Fabry's encephalopathy. It is important to focus on neurological symptoms and suspect Fabry's Disease disease as early as possible and evadeto avoid irreversible damage.
\end{abstract}

Keywords: Fabry disease, Neurological complications, Stroke

La malattia di Fabry (MF) è una malattia rara con dati contrastanti sulla reale incidenza e prevalenza, ma sicuramente è spesso vista ma poco diagnosticata e i primi sintomi clinici, anche di tipo neurologico, vengono frequentemente sottovalutati o trattati come singoli disturbi. La malattia coinvolge sia il sistema nervoso periferico che il sistema nervoso centrale con uno spettro sindromico eterogeneo, dove è comunque possibile individuare alcune costanti sulla modalità di insorgenza dei sintomi neurologici. I pazienti con MF già in giovane età (in media 11 anni) tipicamente manifestano una neuropatia dolorosa (1) al punto da considerare il dolore neuropatico e le acroparestesie come uno dei principali segnali di sospetto diagnostico (2-4). II 70-80\% dei pazienti FD manifesta dolore neuropatico e a lamentarlo sono soprattutto i pazienti maschi, meno le donne, localizzato alle estremità degli arti, palmo delle mani e pianta dei piedi, anche se può localizzarsi in qualsiasi altra parte del corpo mimando a volte una malattia reumatica. I sintomi possono essere presenti a riposo o sono tipicamente accentuati e/o scatenati dalle brusche variazioni della temperatura corporea o della temperatura dell'ambiente esterno e col tempo tendono ad autolimitarsi. II 15-30\% dei pazienti lamentano dolore al collo e mal di testa non sempre distinguibile dalla classica cefalea $(5,6)$. Il dolore neuropatico è dovuto al malfunzionamento delle fibre nervose di piccolo calibro, al depauperamento delle terminazioni nervose intraepidermiche e al danno endoteliale dei vasa nervorum.

Il coinvolgimento del Sistema Nervoso Autonomo è frequen-

Accepted: July 30, 2017

Published online: August 31, 2017

Indirizzo per la corrispondenza:

Dr. Antonello Giordano

UOC Neurologia

P.O. "R. Guzzardi"

Via Papa Giovanni XXIII

97019 Vittoria (RG)

antonello.giordano@asp.rg.it te e sottovalutato e si manifesta con sintomi variegati. I sintomi gastrointestinali, riportati in circa il $60 \%$ dei pazienti con FD, sono i dolori addominali e la diarrea, mentre meno comuni appaiono la nausea, il vomito e la sazietà precoce (7). L'età media di insorgenza di questi sintomi è 14 anni. La sintomatologia è dovuta all'accumulo del GL3 nelle cellule muscolari lisce, endoteliali e dei gangli dorsali (8). Un altro sintomo del coinvolgimento del Sistema Nervoso Autonomo è l'alterazione della sudorazione che si manifesta con l'ipoidrosi o l'anidrosi. Il conseguente difetto di termoregolazione porta a un difetto di termoregolazione con intolleranza all'aumento della temperatura corporea e all'aumento delle temperature ambientali e la cui causa potrebbe essere la compromessa innervazione delle ghiandole sudoripare.

L'ipoacusia neurosensoriale rappresenta un'altra importante manifestazione della malattia. La perdita di udito acuta è 60 volte più frequente nella MF rispetto alla popolazione normale (9). La perdita di udito è solitamente lenta e progressiva e interessa entrambe le orecchie. L'insorgenza avviene nella seconda decade di vita negli uomini e nella quarta decade nelle donne. Inoltre, il disturbo riguarda principalmente le frequenze alte rispetto alle frequenze più basse. Probabilmente la perdita di udito cronica è il risultato dell'accumulo di Gb3 nei gangli audio vestibolari e nei vasi della coclea (10).

II deposito di Gb3 nelle leptomeningi, nelle cellule neuronali e nelle cellule endoteliali di tutto il letto vascolare cerebrale determina il coinvolgimento del Sistema Nervoso Centrale (SNC) nella MF. II 70\% dei pazienti presenta manifestazioni cliniche e neuroradiologiche di malattia cerebrovascolare a dimostrazione di quanto sia rilevante tale aspetto e gli eventi cerebrovascolari ischemici (infarti lacunari o regionali e TIA) sono i più frequenti, coinvolgendo il $25 \%$ dei pazienti con FD. Le lesioni ischemiche interessano tutto l'encefalo anche se con maggior frequenza si ritrovano nei territori del circolo posteriore. Dissecazioni carotidee, emorragie cerebrali intraparenchimali e subaracnoidee, microbleeding e trombosi venosa cerebrale sono gli altri eventi cerebrovascolari descritti nei pazienti con FD. Particolare attenzione è 
stata posta sull'ictus ischemico giovanile criptogenetico e MF quale causa dell'evento cerebrovascolare con la conferma dei dati di letteratura che è una causa da tenere sempre in considerazione nei casi di ictus ischemico giovanile ( $<55 / 60$ anni) dove la FD è presente con una frequenza inferiore all' $1 \%$ (11). Gli studi di neuroimaging hanno evidenziato come il danno a carico dei vasi cerebrali di piccolo e grosso calibro si manifesti con la dolicoectasia dei vasi arteriosi vertebrobasilari e in particolare dell'arteria basilare $(12,13)$, evidenziando un diametro basilare significativamente aumentato nei maschi con FD e con una sofferenza della sostanza bianca cerebrale per la presenza di infarti lacunari multipli cerebrali periventricolari e cerebellari, nei nuclei della base e nel tronco. Secondo alcuni lavori le manifestazioni cerebrovascolari sarebbero una modalità specifica di presentazione della malattia e sono più frequenti di quanto riconosciuto. Altri ricercatori sottolineano, invece, come il danno d'organo sul sistema cerebrovascolare debba essere considerato il risultato finale determinato dalla somma di differenti fattori patogenetici, genetici e acquisiti. Pertanto rimane ancora da chiarire se il danno al SNC sia indipendente dal coinvolgimento di altri organi (aritmie e fibrillazione atriale) e da fattori individuali (ipertensione) o sia determinato da una multifattorietà di componenti. Un cenno merita il danno della barriera emato-encefalica documentato nei pazienti con malattia di Fabry che determina un aumento delle proteine nel liquor dovuto a un interessamento diretto delle meningi e dei vasi meningei.

Più recente è, invece, l'interesse per gli aspetti cognitivi e psicologici nella MF. I pochi studi presenti in letteratura hanno evidenziato diverse discrepanze in merito al coinvolgimento delle funzioni cognitive nei soggetti con malattia di Fabry. Inoltre, l'uso di strumenti differenti lascia aperta la questione della confrontabilità dei dati raccolti. Secondo alcuni autori (14-16) non è possibile parlare di un profilo neuropsicologico specifico del paziente con FD in quanto dagli studi effettuati tali pazienti non presentavano performance cognitive significativamente differenti rispetto ai controlli. Diversamente, altri autori hanno elaborato un profilo psichiatrico e cognitivo specifico che comporta un coinvolgimento neurocognitivo. I risultati di Segal et al (17), per esempio, mettono in evidenza prestazioni nella norma nella maggior parte delle funzioni cognitive indagate con selettiva compromissione delle funzioni esecutive e della velocità di elaborazione delle informazioni. Della stessa opinione è il lavoro di Elstein et al (18) dove i ricercatori segnalano la presenza di un lieve declino cognitivo, non associato a danno vascolare, i cui domini cognitivi maggiormente compromessi sembrano essere quelli adibiti alla velocità di processamento delle informazioni. Altro aspetto interessante ma ancora controverso è l'impatto psicologico che la malattia di Fabry ha sul paziente affetto e sicuramente l'ansia e la depressione sono i sintomi psicologici più frequenti, ma quanto questi sono correlabili al danno neurologico?

\section{Disclosures}

Financial support: No financial support was received for this submission.

Conflict of interest: The author has no conflict of interest.

\section{Bibliografia}

1. Politei JM, Bouhassira D, Gerrmain DP, et al. Pain in Fabry disease: practical recommendations for diagnosis and treatment. CNS Neurosci Ther. 2016;22(7):568-76.

2. Schiffmann R, Scott LJ. Pathophysiology and assessment of neuropathic pain in Fabry disease. Acta Paediatr Suppl. 2002; 91(439):48-52.

3. Moller AT, Jensen TS. Neurological manifestations in Fabry's disease. Nat Clin Pract Neurol. 2007;3(2):95-106.

4. Tuttolomondo A, Pecoraro R, Simonetta I, et al. Neurological complications of Anderson-Fabry disease. Curr Pharm Des. 2013;19(33):6014-30.

5. Burlina AP, Sims KB, Politei JM, et al. Early diagnosis of peripheral nervous system involvement in Fabry disease and treatment of neuropathic pain: the report of an expert panel. BMC Neurol. 2011;11:61.

6. Liguori R, Di Stasi V, Bugiardini E, et al. Small fiber neuropathy in female patients with Fabry disease. Muscle Nerve. 2010; 41(3):409-12.

7. Zar-Kessler C, Karaa A, Sims KB, Clarke V, Kuo B. Understanding the gastrointestinal manifestations of Fabry disease:promoting prompt diagnosis. Therap Adv Gastroenterol. 2016;9(4): 626-34.

8. Pensabene L, Sestito S, Nicoletti A, Graziano F, Strisciuglio $P$, Concolino D. Gastrointestinal Symptoms of Patients with Fabry Disease. Gastroenterol Res Pract. 2016;2016:9712831.

9. Ries M, Kim HJ, Zalewski CK, et al. Neuropathic and cerebrovascular correlates of hearing loss in Fabry disease. Brain. 2007;130(Pt 1):143-50.

10. Palla A, Hegemann S, Widmer U, Straumann D. Vestibular and auditory deficits in Fabry disease and their response to enzyme replacement therapy. J Neurol. 2007;254(10):1433-42.

11. Rolfs A, Fazekas F, Grittner U, et al. Stroke in Young Fabry Patients (Sifap) Investigators. Acute cerebrovascular disease in the young: the Stroke in Young Fabry Patients study. Stroke. 2013;44(2):340-9.

12. Buechner $\mathrm{S}$, Moretti $\mathrm{M}$, Burlina $\mathrm{AP}$, et al. Central nervous system involvement in Anderson-Fabry disease: A clinical and MRI retrospective study. J Neurol Neurosurg Psychiatry. 2008;79(11):1249-54.

13. Borsini W. Il sistema nervoso e la malattia di Fabry. Therapy Perspectives. 2010;XII(2):3-32.

14. Wadley VG, McClure LA, Warnock DG, et al. Cognitive function in adults aging with Fabry disease: a case-control feasibility study using telephone-based assessment. JIMD Rep. 2015;18:41-50.

15. Fellgiebel A, Wolf DO, Kolodny E, Muller MJ. Hippocampal atrophy as a surrogate of neuronal involvement in Fabry disease. J Inherit Metab Dis. 2012;35(2):363-7.

16. Low M, Nicholls K, Tubridy N, et al. Neurology of Fabry disease. Intern Med J. 2007;37(7):436-47.

17. Segal P, Kohn Y, Pollak Y, et al. Psychiatric and cognitive profile in Anderson-Fabry patients: a preliminary study. J Inherit Metab Dis. 2010;33(4):429-36.

18. Elstein D, Doniger GM, Altarescu G. Cognitive testing in Fabry disease: pilot using a brief computerized assessment tool. Isr Med Assoc J. 2012;14(10):624-8. 